Proc. 15th Int. Conference on Defects Recognition, Imaging and Physics in Semiconductors, Warsaw, Poland 2013

\title{
Dislocation Generation and Propagation across the Seed in Seed Cast-Si Ingots
}

\author{
Y. Miyamura*, J. Chen, R.R. Prakash, K. Jiptner, H. Harada and T. Sekiguchi \\ MANA Nanoelectronic Materials Unit, National Institute for Materials Science \\ 1-1 Namiki, Tsukuba, Ibaraki 305-0044, Japan
}

\begin{abstract}
We have studied the dislocation generation and propagation from the seed crystals during seed cast Si growth. The grown ingot was cut into a vertical wafer, followed by the dislocation imaging using X-ray topography and Secco etching. The dislocation behavior at the seed area was compared with the dislocation generation at the top surface due to the thermal stress during cooling. The dislocations at the seed/crystal interface have propagated on the $\{111\}$ plane toward top. When the seed surface was not melted sufficiently, the interface defect density became high, but no clear dislocation propagation was recognized. This suggests that the thermal shock at the seed $/ \mathrm{melt}$ interface was not high enough to propagate dislocations to the growth direction. A certain amount of dislocations has been introduced from the top into the ingot according to the thermal stress. These observations suggest that optimizing the initial growth condition is important to dislocation control.
\end{abstract}

DOI: 10.12693/APhysPolA.125.1024

PACS: 88.40.jj, 81.10.Fq, 61.72.Ff, 61.72.Hh, 61.72.Lk

\section{Introduction}

The seed cast method has attracted much attention for the growth of monocrystalline (mono-) Si, because mono-Si solar cell is expected to possess high efficiency of single crystalline $\mathrm{Si}$ and low cost of multicrystalline Si. In 2008, BP solar group has demonstrated the seed cast growth technique [1, 2]. Although mono-Si ingot should be free from grain boundaries, they often contain certain densities of dislocation and lineage. Most of seed cast techniques adopt the multi seeds to cover all the bottom of crucible and perform one-directional solidification. However, this method inevitably introduces small angle grain boundaries (SAGBs) at the edge of the seed wafer. Thus, we have developed the technique using single small seed to grow ingot three dimensionally. We have named it Mushroom growth.

This method has an advantage of the crystallinity because the ingots are free from the SAGBs generated at the seed edges, although proper growth condition is necessary to produce the ingot with low defect density. In this method most of the extended defects are propagated from the seed. Thus, it is necessary to minimize such dislocation propagation from the seed. This paper discusses such defect propagation phenomena by using X-ray topography and etch pit observation.

\section{Experimental}

Mono-Si ingots were grown by seed cast technique using a laboratory furnace and a pilot furnace. Using the former furnace we can grow $10 \mathrm{~cm}$ diameter ingot from

*corresponding author; e-mail: miyamura.yoshiji@nims.go.jp the same size seed [3-5] and using the latter we can get $50 \mathrm{~cm}$ square from $20 \mathrm{~cm}$ diameter seed. In both cases, the seed crystals were cut from $\mathrm{Cz}$ (100) ingot. First the growth condition was optimized using the former. Then, the Mushroom growth was realized using the latter [6-8].

The dislocation propagation was elucidated using X-ray topography (XRT) and etch pit observation using Secco etchant.

\section{Results and discussion}

Figure 1 shows the XRT image of vertically sliced wafer from $10 \mathrm{~cm}$ diameter seed-cast ingot. This XRT image is taken at $g=400$ reflection. Since the twinning has taken place at the middle stage of crystal growth, the upper area appears dark. The white region at the bottom is the seed crystal, indicating that the dislocation networks are developed. Although the original seed crystal is free from dislocations, the thermal stress during the growth may introduce dislocations. Nevertheless, the area just above the seed is darker, indicating that most of the dislocations at the seed have not propagated to the growth area. After about the $10 \mathrm{~mm}$ growth, as shown with arrows, the white dots have appeared. Some dislocations have generated from these dots. They are the light element precipitates such as $\mathrm{Si}_{3} \mathrm{~N}_{4}$ or $\mathrm{SiC}$. Long straight lines correspond to slip dislocations or twin lamellae.

Figure 2 shows two XRT images of the $10 \mathrm{~cm}$ seed-cast Si samples. Those images are taken at $g=400$ reflection. These vertical wafers indicate that more than half of seed has been melted.

In Fig. 2a there exist dark bands at the right area of ingot. They are lineage, in which dislocations have bundled and continue to propagate to upper region. There exist short dark lines at the left bottom of ingot. They may be related to the dislocations introduced at the bottom 


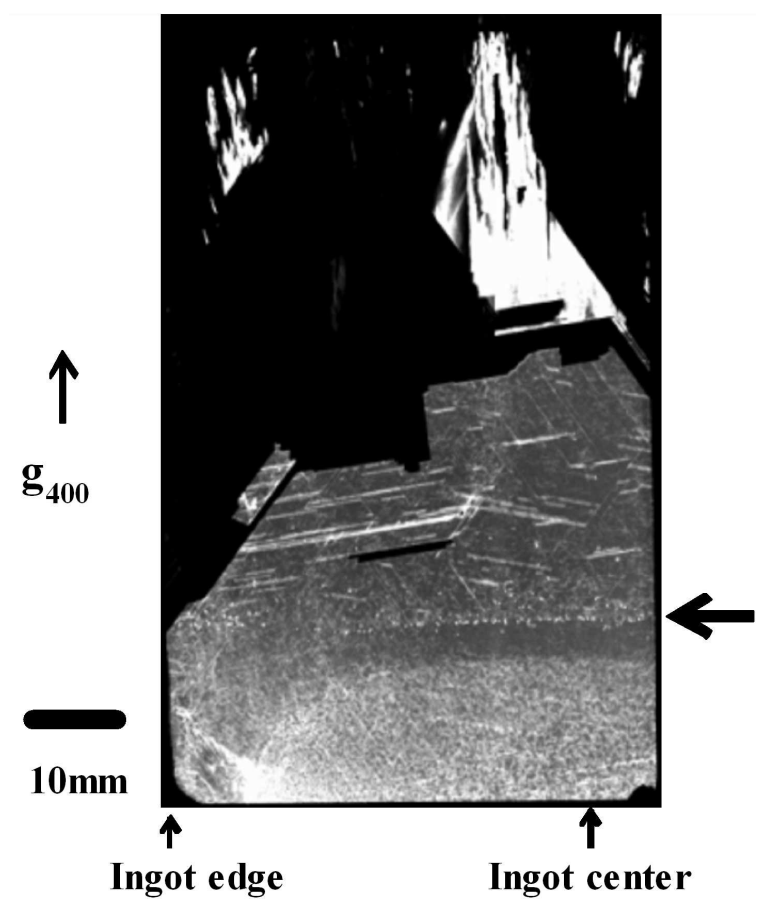

Fig. 1. XRT image of vertically sliced sample from mono-Si.
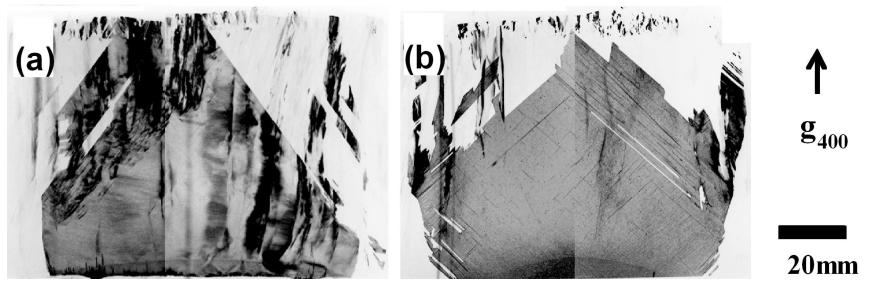

Fig. 2. XRT images of vertically sliced samples from mono-Si.

of the seed crystal. Some stress between the seed bottom and crucible coating may introduce such dislocations.

In Fig. 2b, on the other hand, the ingot looks better although there exist dark lines and dots, which correspond to slip dislocations. Most of the dislocations were introduced in $\{111\}$ planes by slipping. Only one lineage propagates at the right center region. The crystal quality of this ingot is better than the former because no significant dislocation bundle appears. In this ingot, the seed also contains tangled dislocations and some of them are propagated to upper region but terminated. This indicates that they are moved from the seed due to the stress during cooling.

These observations suggest that the proper seed melting and reduction of the thermal stress are the two important ways to reduce extended defects in monocast $\mathrm{Si}$ ingot.

Considering these points, the large ingot was grown in the $50 \mathrm{~cm}$ square furnace. Figure 3 shows the X-ray topography of seed and grown mono-ingot (right half of

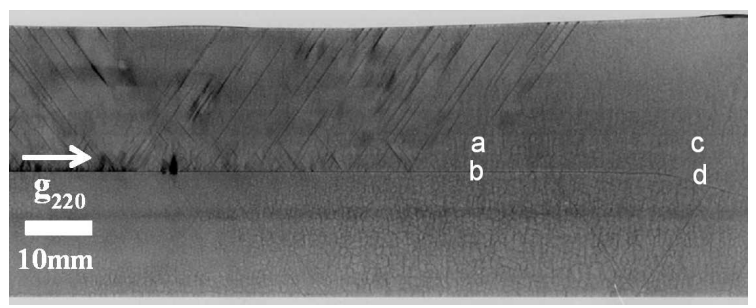

Fig. 3. XRT image of vertically sliced sample from mono-Si.

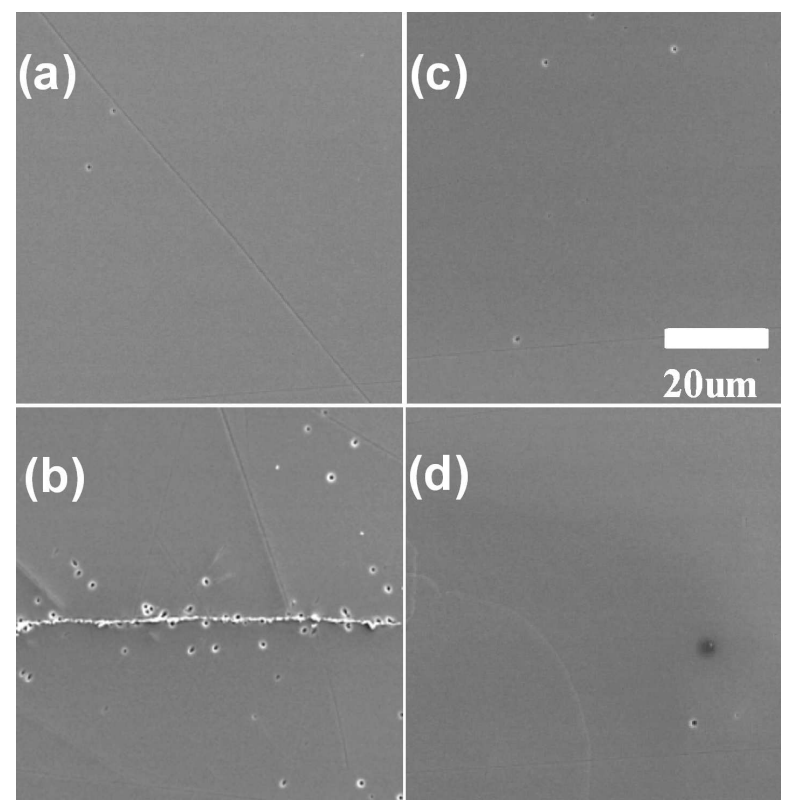

Fig. 4. SEM images after Secco etching of vertically sliced samples from mono-Si.

vertical cut wafer) taken at $g=220$ reflection. The growth is terminated at $50 \mathrm{~mm}$ for checking the dislocation propagation. The interface is clearly distinguished as the middle horizontal line. The lower seed crystal also contains dislocation network, which may be introduced by the thermal stress during the growth. The central side of the upper grown region has straight lines running along two $\{111\}$ planes. They are slip dislocations introduced from the seed/crystal interface and top surface. Such straight dislocations are not observed at the right region. The seed/crystal interface runs downward in this region, suggesting the seed melting. This indicates that when the seed surface is enough melted, the interface does not act as the nucleation site for slip dislocations.

On the other hand, the dislocations introduced from the surface are not observed in this region. It may suggest that the strain during cooling was small compared with the central region. The etching experiment was performed to distinguish the origin of the absence of straight dislocation.

Figure 4 shows the etch pit images of the four regions shown in Fig. 3. The seed/crystal interface at area $b$ is rough and easily distinguished as a groove. This interface 
becomes faint and is only distinguished as the trail of etch pits. At area $d$ where the original seed surface has been melted, only a few etch pits are observed on the interface. The dislocation density at the area $b$ is $7 \times 10^{5} \mathrm{~cm}^{-2}$, while at the area $d$ is $2 \times 10^{4} \mathrm{~cm}^{-2}$. However, the dislocation density $2 \mathrm{~mm}$ above $b$ (area $a$ ) is $3 \times 10^{4} \mathrm{~cm}^{-2}$, while $2 \mathrm{~mm}$ above $d$ (area $c$ ) is $4 \times 10^{4} \mathrm{~cm}^{-2}$. These data suggests that the interfacial dislocation does not dominantly determine the dislocation density in the grown crystal region. Anyway, it is better to prepare smooth interfaces to avoid extra dislocation generation source. The partial melting of the seed surface is preferable for the actual seed growth.

\section{Summary}

In the seed-cast mono-Si growth technique, the defect propagation from the seed was studied by XRT and etch pit observation. The seed crystal often has dislocation network due to the thermal stress during crystal growth and cooling. They are not so detrimental to propagate the grown crystal region. Although it is not clearly demonstrated, the preparation of the smooth interface between seed and grown crystal is preferable to reduce the dislocation propagation.

\section{Acknowledgments}

We thank Dr. T. Tachibana and Prof. A. Ogura (Meiji University), Mr. T. Kojima and Prof. Y. Ohshita (Toyota Technological Institute), Mr. S. Nakano and Prof. K. Kakimoto (Kyushu University) for crystal growth and useful discussion. This work was partly supported by the New Energy and Industrial Technology Development Organization (NEDO) under the Ministry of Economy, Trade and Industry (METI), Japan.

\section{References}

[1] B. Wu, N. Stoddard, R. Ma, R. Clark, J. Cryst. Growth 310, 2178 (2008).

[2] N. Stoddard, B. Wu, I. Witting, M. Wagner, Y. Park, G. Rozgonyi, R. Clark, Solid State Phenom, 131133, 1 (2008).

[3] Y. Miyamura, H. Harada, K. Jiptner, J. Chen, R.R. Prakash, J.Y. Li, T. Sekiguchi, T. Kojima, Y. Ohshita, A. Ogura, M. Fukuzawa, S. Nakano, B. Gao, K. Kakimoto, Solid State Phenom., 205206, 89 (2013).

[4] K. Jiptner, M. Fukuzawa, Y. Miyamura, H. Harada, K. Kakimoto, T. Sekiguchi, Phys. Status Solidi C 10, 141 (2013).

[5] T. Tachibana, T. Sameshima, T. Kojima, K. Arafune, K. Kakimoto, Y. Miyamura, H. Harada, T. Sekiguchi, Y. Ohshita, A. Ogura, J. Appl. Phys. 111, 074505 (2012).

[6] B. Gao, S. Nakano, H. Harada, Y. Miyamura, T. Sekiguchi, K. Kakimoto, J. Cryst. Growth 352, 47 (2012).

[7] B. Gao, S. Nakano, H. Harada, Y. Miyamura, T. Sekiguchi, K. Kakimoto, Cryst. Growth Des. 12 , 6144 (2012).

[8] Y. Miyamura, H. Harada, K. Jiptner, J. Chen, R.R. Prakash, S. Nakano, B. Gao, K. Kakimoto, T. Sekiguchi, to be published in J. Cryst. Growth. 